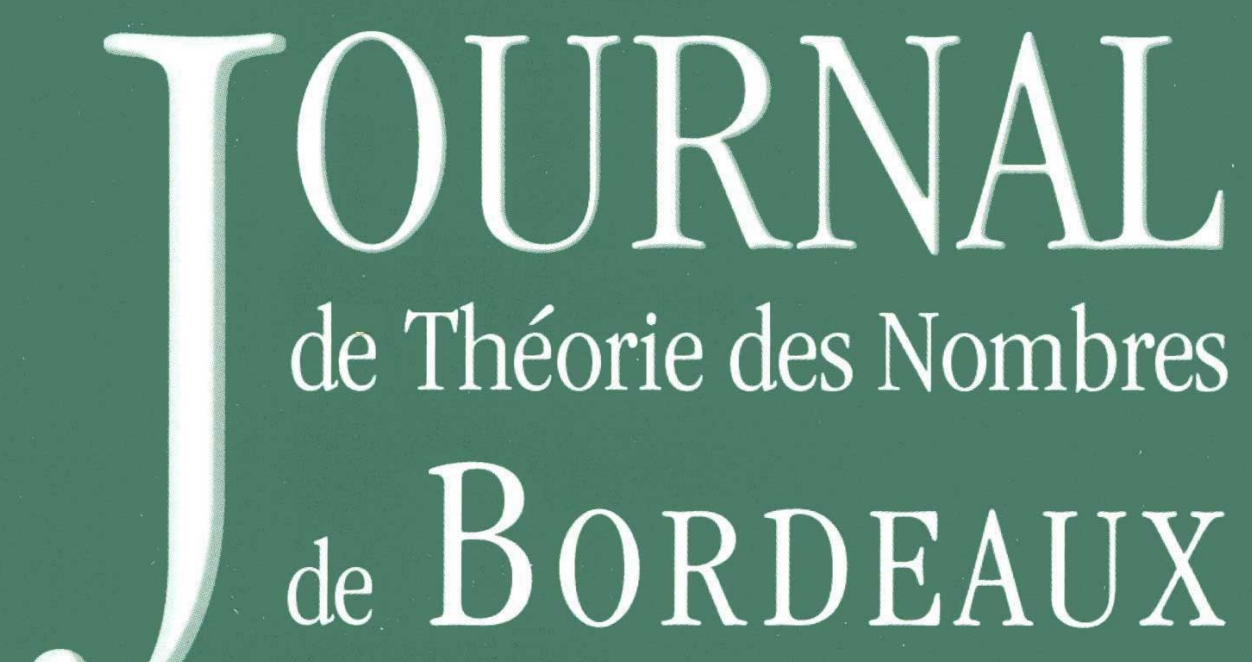

anciennement Séminaire de Théorie des Nombres de Bordeaux

Xavier GUITART et Jordi QUER

\title{
Remarks on strongly modular Jacobian surfaces
}

Tome 23, no 1 (2011), p. 171-182.

<http://jtnb.cedram.org/item?id=JTNB_2011__23_1_171_0>

(C) Société Arithmétique de Bordeaux, 2011, tous droits réservés.

L'accès aux articles de la revue «Journal de Théorie des Nombres de Bordeaux » (http://jtnb.cedram.org/), implique l'accord avec les conditions générales d'utilisation (http://jtnb.cedram. org/legal/). Toute reproduction en tout ou partie cet article sous quelque forme que ce soit pour tout usage autre que l'utilisation à fin strictement personnelle du copiste est constitutive d'une infraction pénale. Toute copie ou impression de ce fichier doit contenir la présente mention de copyright.

\section{cedram}




\title{
Remarks on strongly modular Jacobian surfaces
}

\author{
par XAVIER GUITART et Jordi QUER
}

\begin{abstract}
RÉsumÉ. Dans [3] nous avons introduit la notion de variété abélienne fortement modulaire. Cette note contient quelques remarques et des exemples de ce type de variétés, surtout pour le cas des surfaces Jacobiennes, qui complètent les résultats de [3].
\end{abstract}

ABSTRACT. In [3] we introduced the concept of strongly modular abelian variety. This note contains some remarks and examples of this kind of varieties, especially for the case of Jacobian surfaces, that complement the results of [3].

\section{Introduction}

One of the most impressive achievements of the last years in number theory is the proof of the modularity of all rational elliptic curves by Breuil, Conrad, Diamond and Taylor, following the ideas and techniques introduced by A. Wiles. This fact, among several other equivalent ways, can be stated as follows: every elliptic curve $A / \mathbb{Q}$ defined over the rational numbers is $\mathbb{Q}$-isogenous to a simple factor of some variety $J_{1}(N)$.

From Serre's conjecture on modularity of $\bmod p$ two-dimensional Galois representations, recently proven by Khare and Winterberger, one obtains (cf. Ribet [8]) the more general characterization of the $\mathbb{Q}$-simple varieties $A / \mathbb{Q}$ that are $\mathbb{Q}$-isogenous to a simple factor of some variety $J_{1}(N)$. They are the varieties called of of $\mathrm{GL}_{2}$-type, defined by the condition that $\operatorname{End}_{\mathbb{Q}}^{0}(A)$ is a number field of degree equal to $\operatorname{dim} A$. Much of the interest of these varieties lies in the fact that their $L$-function is equivalent to a product of $L$-functions of classical modular forms for congruence subgroups $\Gamma_{1}(N)$. In addition, if a $\mathbb{Q}$-simple variety has this property then it is of $\mathrm{GL}_{2}$-type.

In [5] Pyle characterizes the abelian varieties $B / K$ defined over a number field $K$ that are simple factors of some abelian variety of $\mathrm{GL}_{2}$-type. Equivalently, thanks to the modularity result stated in the previous paragraph, this is a characterization of the the absolutely simple factors up to isogeny of modular Jacobians $J_{1}(N)$. She calls them building blocks. The non-CM building blocks are precisely the varieties admitting compatible

Research of both authors supported by grants 2009SGR-1220 and MTM2009-13060-C02-01. 
isogenies between their Galois conjugates, and whose endomorphism algebra has totally real center and has reduced degree equal to the dimension of the variety.

In [3] we introduced the concept of strongly modular abelian variety as a variety $B / K$ over a number field $K$ whose $L$-function $L(B / K ; s)$ is equivalent to a product of $L$-functions of classical modular forms for congruence subgroups $\Gamma_{1}(N)$. This property is characterized in [3, Theorem 5.3], with the additional hypothesis of $K / \mathbb{Q}$ being a Galois extension, in terms of the existence of compatible isogenies between Galois conjugates, the structure of the endomorphism algebra of the variety, and properties of a certain Galois cohomology class $\left[c_{B / K}\right]$ attached to it.

In the present paper we continue the study of strongly modular abelian varieties by complementing the results of [3] in three different aspects. First of all, in Section 2 we prove that if the variety $B / K$ is strongly modular then the number field $K$ must necessarily be Galois (and abelian) over $\mathbb{Q}$; this shows that the Galois condition can be removed from the hypothesis of the main theorem [3, Theorem 5.3]. The proof is obtained from an elementary general result (Lemma 2.1) about the field of definition of endomorphisms of abelian varieties obtained by restriction of scalars, and the use of Ribet's results on twists of modular forms. Second, in [3] the cohomology classes $\left[c_{B / K}\right]$ were explicitly computed for the Jacobians of a certain family of genus two curves. In Section 3 we extend this computation to the Jacobians of a much wider class of genus two curves investigated by Rotger in [10]. Finally, in Section 4, we make a detailed study of an example that was already mentioned in [3]: it is an abelian surface $B / K$ defined over a biquadratic field $K=\mathbb{Q}(\sqrt{2}, \sqrt{-3})$ as the Jacobian of an explicitly given hyperelliptic curve $C / K$. It is a building block but it is not strongly modular over $K$; moreover, none of its twists is strongly modular over $K$. What we do is to show how to twist the defining curve $C$ in such a way that the corresponding Jacobian is a strongly modular surface, but over the bigger field $K(\sqrt{-1})$.

\section{Restriction of scalars and $\boldsymbol{L}$-series of building blocks}

We begin by recalling the basic definitions and properties of the objects we will be working with; more details can be found in [3].

An abelian $\mathbb{Q}$-variety is an abelian variety $B / \overline{\mathbb{Q}}$ with the property that for each $\sigma \in G_{\mathbb{Q}}=\operatorname{Gal}(\overline{\mathbb{Q}} / \mathbb{Q})$ there exists an isogeny $\mu_{\sigma}:{ }^{\sigma} B \rightarrow B$ compatible with the endomorphisms of $B$; that is, such that $\varphi \circ \mu_{\sigma}=\mu_{\sigma}{ }^{\circ}{ }^{\sigma} \varphi$ for all $\varphi \in \operatorname{End}^{0}(B)$. A building block is an abelian $\mathbb{Q}$-variety such that $\operatorname{End}^{0}(B)$ is a division algebra of Schur index $t \leqslant 2$ whose center is a totally real number field $F$ and $t[F: \mathbb{Q}]=\operatorname{dim} B$. The building blocks are the non-CM $\overline{\mathbb{Q}}$-simple 
factors of the varieties of $\mathrm{GL}_{2}$-type (this is $[5, \S 4]$ ), and therefore they are the absolutely simple quotients without CM of the varieties $J_{1}(N)_{\overline{\mathbb{Q}}}$.

Let $B$ be a building block, and let $F$ be the center of its endomorphism algebra. Let $K / \mathbb{Q}$ be a Galois extension with Galois group $G$. We will say that $B$ is completely defined over $K$ if $B$, its endomorphisms and all the isogenies between $B$ and its conjugated varieties are defined over $K$. If this is the case, for each $s \in G$ fix an isogeny $\mu_{s}:{ }^{s} B \rightarrow B$, and for $s, t \in G$ define $c_{B / K}(s, t)=\mu_{s}{ }^{\circ}{ }^{s} \mu_{t}{ }^{\circ} \mu_{s t}^{-1}$. This map is a two-cocycle of $G$ with values in the $G$-module with trivial action $F^{\times}$. Its cohomology class $\left[c_{B / K}\right]$ belongs to $H^{2}\left(G, F^{\times}\right)$, it is independent of the choices of the $\mu_{s}$ and it is an invariant of the $K$-isogeny class of $B$; moreover, it is a 2-torsion element. Let $c_{B}$ be the inflation of $c_{B / K}$ to $G_{\mathbb{Q}}$; its cohomology class $\left[c_{B}\right]$ belongs to $H^{2}\left(G_{\mathbb{Q}}, F^{\times}\right)[2]$ ( $F^{\times}$with trivial $G_{\mathbb{Q}^{-a c t i o n}}$ ) and it is an invariant of the $\overline{\mathbb{Q}}$-isogeny class of $B$.

By considering an embedding of $F$ into $\mathbb{R}$ one obtains a decomposition of $H^{2}\left(G_{\mathbb{Q}}, F^{\times}\right)[2]$ as

$$
H^{2}\left(G_{\mathbb{Q}}, F^{\times}\right)[2] \simeq H^{2}\left(G_{\mathbb{Q}},\{ \pm 1\}\right) \times \operatorname{Hom}\left(G_{\mathbb{Q}}, F^{\times} /\{ \pm 1\} F^{\times 2}\right) .
$$

The first component of $\left[c_{B}\right]$ under this isomorphism, that we will denote $\left[c_{B}\right]_{ \pm}$, is obtained by taking the sign of $c_{B}$. We will use that $H^{2}\left(G_{\mathbb{Q}},\{ \pm 1\}\right)$ is canonically isomorphic to the 2-torsion of the Brauer group of $\mathbb{Q}$, and we will identify $\left[c_{B}\right]_{ \pm}$with a rational quaternion algebra. The second component, that we will denote $\overline{\left[c_{B}\right]}$, is given by a coboundary of $c_{B}^{2}$ : if $\lambda: G_{\mathbb{Q}} \rightarrow F^{\times}$ is a map such that $c_{B}^{2}(\sigma, \tau)=\lambda(\sigma) \lambda(\tau) \lambda(\sigma \tau)^{-1}$ then the homomorphism $G \rightarrow F^{\times} /\{ \pm 1\} F^{\times 2}: \sigma \mapsto \lambda(\sigma)\{ \pm 1\} F^{\times 2}$ is precisely $\overline{\left[c_{B}\right]}$. In practice, a coboundary of $c_{B}^{2}$ can be computed by means of the degree map: fix $\rho: B \rightarrow \hat{B}$ a polarization of $B$, and for each $\sigma \in G_{\mathbb{Q}}$ fix a compatible isogeny $\mu_{\sigma}:{ }^{\sigma} B \rightarrow B$ and define the degree of $\mu_{\sigma}$ by $d\left(\mu_{\sigma}\right)=\mu_{\sigma}{ }^{\sigma}{ }^{\sigma} \rho^{-1} \circ \hat{\mu}_{\sigma}{ }^{\circ} \rho$, which is an element of $F^{\times}$. Then $c_{B}^{2}(\sigma, \tau)=d\left(\mu_{\sigma}\right) d\left(\mu_{\tau}\right) d\left(\mu_{\sigma \tau}\right)^{-1}$, so the component $\overline{\left[c_{B}\right]}$ is the map $d: \sigma \mapsto d\left(\mu_{\sigma}\right)\{ \pm 1\} F^{\times}$.

Let $B$ be a building block defined over a number field $K$, and let $F$ be the center of $\operatorname{End}^{0}(B)$. For simplicity suppose that all the endomorphisms of $B$ are defined over $K$ (see however remark 2.4). The $L$-series $L(B / K ; s)$ is equivalent to a product of $L$-series of modular forms for $\Gamma_{1}(N)$ if and only if the restriction of scalars $\operatorname{Res}_{K / \mathbb{Q}} B$ is isogenous over $\mathbb{Q}$ to a product of abelian varieties of $\mathrm{GL}_{2}$-type (see [3, Proposition 2.4] for the details). In the case where $K / \mathbb{Q}$ is Galois, a necessary and sufficient condition for this was given in $[3, \S 5]$ in terms of the cohomology class $\left[c_{B / K}\right]$. Now we show that, in fact, if $\operatorname{Res}_{K / \mathbb{Q}} B$ is isogenous to a product of varieties of $\mathrm{GL}_{2}$-type then $K / \mathbb{Q}$ is necessarily a Galois extension. This will be a consequence of the following 
Lemma 2.1. Let $k$ be a field and $\bar{k}$ a separable closure. Let $K, L$ be subfields of $\bar{k}$ of finite degree over $k$. Let $B$ be an abelian variety over $K$. If the endomorphisms of the variety $A=\operatorname{Res}_{K / k} B$ are defined over $L$, then $K \subseteq$ $L$.

Proof. We will see that $G_{L} \subseteq G_{K}$. Suppose this is not the case. Then there exists an automorphism $\sigma \in G_{L}$ which does not belong to $G_{K}$. Let $\Sigma_{K}$ denote the set of $k$-embeddings $\tau: K \rightarrow \bar{k}$. If we denote by $\tau_{0}$ the inclusion $\tau_{0}: K \hookrightarrow \bar{k}$, then $\sigma \tau_{0} \neq \tau_{0}$. We will construct an element $\varphi \in \operatorname{End}_{\bar{k}}(A)$ such that ${ }^{\sigma} \varphi \neq \varphi$, and this will be a contradiction with the fact that $\operatorname{End}_{\bar{k}}(A)=\operatorname{End}_{L}(A)$.

Let $A_{0}$ be the variety $A_{0}=\prod_{\tau \in \Sigma_{K}}{ }^{\tau} B$, and for each $\rho \in G_{k}$ let $\phi_{\rho}:{ }^{\rho} A_{0} \rightarrow A_{0}$ be the isomorphism which permutes the coordinates according to the canonical isomorphisms ${ }^{\rho}\left({ }^{\tau} B\right) \simeq{ }^{\rho \tau} B$. By the construction of the variety restriction of scalars, there exists a $\bar{k}$-isomorphism $\lambda: A_{0} \rightarrow A$ such that $\lambda^{-1}{ }^{\rho} \lambda=\phi_{\rho}(\mathrm{cf} .[11, \S 1.3])$.

Let $\psi$ be a diagonal endomorphism of $A_{0}$; that is, one of the form $\prod_{\tau \in \Sigma_{K}} \psi_{\tau}$, with each $\psi_{\tau}$ an element of $\operatorname{End}\left({ }^{\tau} B\right)$. Since $\sigma \tau_{0} \neq \tau_{0}$ we can, and do, choose such a $\psi$ with ${ }^{\sigma} \psi_{\tau_{0}} \neq \psi_{\sigma \tau_{0}}$. Consider now the endomorphism of $A$ given by $\varphi=\lambda \circ \psi \circ \lambda^{-1}$. If $\sigma_{\varphi}=\varphi$ then $\lambda^{-1}{ }_{\circ}{ } \lambda_{\circ} \sigma \psi=\psi \circ \lambda^{-1}{ }_{\circ} \sigma \lambda$, and therefore $\phi_{\sigma}{ }^{\circ}{ }^{\sigma} \psi=\psi \circ \phi_{\sigma}$. But the restriction of $\phi_{\sigma}{ }^{\circ}{ }^{\sigma} \psi$ to ${ }^{\sigma \tau_{0}} B$ is equal to ${ }^{\sigma} \psi_{\tau_{0}}$, whereas the restriction of $\psi \circ \phi_{\sigma}$ to ${ }^{\sigma \tau_{0}} B$ is equal to $\psi_{\sigma \tau_{0}}$. But we chose $\psi$ with ${ }^{\sigma} \psi_{\tau_{0}} \neq \psi_{\sigma \tau_{0}}$, so ${ }^{\sigma} \varphi \neq \varphi$ and therefore not all the endomorphisms of $A$ are defined over $L$; this is a contradiction, thus $G_{L}$ is indeed contained in $G_{K}$ as we aimed to see.

Proposition 2.2. Let $B$ be a building block defined over a number field $K$. If $\operatorname{Res}_{K / \mathbb{Q}} B$ is isogenous over $\mathbb{Q}$ to a product of abelian varieties of $\mathrm{GL}_{2}$-type without $C M$, then the extension $K / \mathbb{Q}$ is abelian.

Proof. Since each abelian variety of $\mathrm{GL}_{2}$-type is isogenous over $\mathbb{Q}$ to a modular abelian variety, we can suppose that $A$ is isogenous over $\mathbb{Q}$ to a product of the form $\prod_{f} A_{f}$, for some weight two newforms $f$. The minimal field of definition of the endomorphisms of $A_{f}$ is an abelian extension $L_{f} / \mathbb{Q}$ by [2, Proposition 2.1]. On the other hand, suppose that $A_{f}$ and $A_{g}$ are simple factors over $\mathbb{Q}$ of $\operatorname{Res}_{K / \mathbb{Q}} B$ such that $\operatorname{Hom}_{\overline{\mathbb{Q}}}\left(A_{f}, A_{g}\right) \neq 0$. Then by $[9$, Theorem 4.7] we can suppose that there exists a Dirichlet character $\chi$ such that $f=g \otimes \chi$. If we identify $\chi$ with a Galois character $\chi: G_{\mathbb{Q}} \rightarrow \overline{\mathbb{Q}}^{\times}$ via class field theory, and we denote by $M$ the fixed field of the kernel of $\chi$, then $\operatorname{Hom}_{M}\left(A_{f}, A_{g}\right) \neq 0$. That is, there exists a homomorphism between $A_{f}$ and $A_{g}$ defined over the abelian extension $M$. This implies that the endomorphisms between $A_{f}$ and $A_{g}$ are defined over the composition $M L_{f} L_{g}$, which is abelian. Thus, all the endomorphisms of $\operatorname{Res}_{K / \mathbb{Q}} B$ are 
defined over a certain abelian extension $L$. Now lemma 2.1 implies that $K \subseteq L$, so that $K$ is also abelian over $\mathbb{Q}$.

Theorem 2.3. Let $B$ be a building block whose endomorphisms are defined over a number field $K$. Then $B$ is strongly modular over $K$ if and only if $K / \mathbb{Q}$ is abelian, $B$ is completely defined over $K$ and $\left[c_{B / K}\right]$ belongs to the subgroup of classes of symmetric cocycles $\operatorname{Ext}\left(G, F^{\times}\right) \subseteq H^{2}\left(G, F^{\times}\right)$.

Proof. Suppose that $L(B / K ; s)$ is a product of $L$-series of modular forms. Then the variety $\operatorname{Res}_{K / \mathbb{Q}} B$ is isogenous over $\mathbb{Q}$ to a product of abelian varieties of $\mathrm{GL}_{2}$-type, and by proposition 2.2 the extension $K / \mathbb{Q}$ is necessarily Galois. Now the result follows from [3, Theorem 5.3].

Remark 2.4. A non-CM abelian variety of $\mathrm{GL}_{2}$-type is isogenous over $\overline{\mathbb{Q}}$ to a power of a building block. Analogously, if $K / \mathbb{Q}$ is a Galois extension with Galois group $G$, then an abelian variety of $\mathrm{GL}_{2}$-type is isogenous over $K$ to a power of a $K$-building block. A $K$-building block is a $\mathbb{Q}$-variety $B$ defined over $K$, with compatible isogenies defined over $K$ and such that the endomorphism algebra $\operatorname{End}_{K}^{0}(B)$ is a central division algebra over a number field $E$, with index $t \leqslant 2$ and $t[E: \mathbb{Q}]=\operatorname{dim} B$. Observe that with this terminology, a building block is the same as a $\overline{\mathbb{Q}}$-building block. To a $K$ building block one also attaches a cohomology class as follows: for each $s \in$ $G$ fix a compatible isogeny $\mu_{s}:{ }^{s} B \rightarrow B$ and define $c_{B / K}(s, t)=\mu_{s}{ }^{s} \mu_{t}{ }^{\circ} \mu_{s t}^{-1}$. Now $c_{B / K}$ is a two cocycle of $G$ with values in $E^{\times}$(endowed with the trivial $G$-action), and its cohomology class $\left[c_{B / K}\right]$ belongs to $H^{2}\left(G, E^{\times}\right)$. Combining proposition 2.2 with [3, Theorem 5.3] as we did in the proof of theorem 2.3 we have the following

Theorem 2.5. Let $B / K$ be a $K$-simple abelian variety. $B$ is strongly modular over $K$ if and only if $K / \mathbb{Q}$ is abelian, $B$ is a $K$-building block and $\left[c_{B / K}\right] \in \operatorname{Ext}\left(G, E^{\times}\right)$.

\section{Cohomology classes attached to Jacobian surfaces with QM}

We begin this section by recalling some notations and results from [10]. For rational numbers $a$ and $b$ we denote by $(a, b)_{\mathbb{Q}}$ the quaternion algebra over $\mathbb{Q}$ generated by $\imath, \jmath$ with $\imath^{2}=a, \jmath^{2}=b$ and $\imath \jmath+\jmath=0$. Let $\mathcal{B}$ be an indefinite quaternion algebra over $\mathbb{Q}$ of discriminant $D>1$. We denote by $\mathrm{n}$ and tr the corresponding reduced norm and trace. Let $\mathcal{O}$ be a maximal order in $\mathcal{B}$. A curve $C / \overline{\mathbb{Q}}$ is said to be a $Q M$-curve with respect to $\mathcal{O}$ if $\mathcal{O}$ can be embedded in the endomorphism ring of its Jacobian.

Fix an element $\mu \in \mathcal{O}$ such that $\mu^{2}+D=0$, whose existence is guaranteed by Eichler's theory of optimal embeddings, and call the pair $(\mathcal{O}, \mu)$ a principally polarized order. A twist of $(\mathcal{O}, \mu)$ is an element $\chi \in \mathcal{O} \cap N_{B^{\times}}(\mathcal{O})$ such that $\chi^{2}+\mathrm{n}(\chi)=0$ and $\chi \mu+\mu \chi=0$, so that $\mathcal{B} \simeq(-D,-\mathrm{n}(\chi))_{\mathbb{Q}}$. The 
pair $(\mathcal{O}, \mu)$ is said to be twisting if it admits some twist in $\mathcal{O}$, and $\mathcal{B}$ is said to be twisting if it contains some twisting polarized maximal order. In fact, $\mathcal{B}$ is twisting if and only if $\mathcal{B} \simeq(-D, m)_{\mathbb{Q}}$ for some integer $m$ dividing $D$.

If $(B, \rho) / \overline{\mathbb{Q}}$ is a polarized abelian variety and $R$ is a subring of $\operatorname{End}(B)$, the field of moduli $k_{R}$ is defined to be the smallest number field such that for any $\sigma \in \operatorname{Gal}\left(\overline{\mathbb{Q}} / k_{R}\right)$ there exists an isomorphism $\phi_{\sigma}:{ }^{\sigma} B \rightarrow B$ with ${ }^{1}$ $\phi_{\sigma}^{*}(\rho)={ }^{\sigma} \rho$ and such that for each $r \in R$ the following diagram commutes:

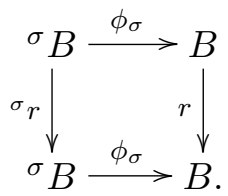

For any curve $C$, we will see its Jacobian as a polarized abelian variety, with the canonical polarization induced by $C$. The field of moduli of $C$, denoted by $k_{C}$, is the smallest number field such that ${ }^{\sigma} C$ and $C$ are isomorphic for all $\sigma \in \operatorname{Gal}\left(\overline{\mathbb{Q}} / k_{C}\right)$. Note that this is the same as the field of moduli $k_{\mathbb{Z}}$ for the subring $\mathbb{Z} \subseteq \operatorname{End}(\operatorname{Jac}(C))$. The following result is $[10$, Theorem 4.1].

Theorem 3.1 (Rotger). Let $C / \overline{\mathbb{Q}}$ be a smooth irreducible curve of genus 2 such that $\operatorname{End}(\operatorname{Jac}(C))$ is isomorphic to a maximal order $\mathcal{O}$ in $\mathcal{B}$. Fix an isomorphism $\mathcal{O} \simeq \operatorname{End}(\operatorname{Jac}(C))$, let $\mu \in \mathcal{O}$ such that $\mu^{2}+D=0$ and suppose that under the previous isomorphism the Rosati involution is given by $\varphi^{\prime}=\mu^{-1} \bar{\varphi} \mu$ for all $\varphi \in \mathcal{O}$, where the bar denotes the canonical involution on $\mathcal{B}$. Suppose that the polarized order $(\mathcal{O}, \mu)$ is twisting and let $m \mid D$ such that $\mathcal{B} \simeq(-D, m)_{\mathbb{Q}}$. Then there exist elements $\omega_{m}$ and $\omega_{D / m}$ belonging to $\mathcal{O}$ such that $\mu \omega_{m}=-\omega_{m} \mu$ and $\mu \omega_{D / m}=-\omega_{D / m} \mu$, with the property that

(1) $\omega_{m}^{2}=m$ and $\omega_{D / m}^{2}=D / m$,

(2) $k_{\mathbb{Z}\left[\omega_{m}\right]}$ and $k_{\mathbb{Z}\left[\omega_{D / m}\right]}$ are at most quadratic extensions of $k_{C}$,

(3) $k_{\mathcal{O}}=k_{\mathbb{Z}\left[\omega_{m}\right]} \cdot k_{\mathbb{Z}\left[\omega_{D / m}\right]}$.

Let $C$ be a curve as in the previous theorem (in particular we continue with the same notation for the elements $\mu, \omega_{m}$ and $\left.\omega_{D / m}\right)$, and let $B$ be its Jacobian. For each $\sigma \in \operatorname{Gal}\left(\overline{\mathbb{Q}} / k_{C}\right)$ the isomorphism ${ }^{\sigma} C \simeq C$ induces an isomorphism of polarized abelian surfaces $\phi_{\sigma}:{ }^{\sigma} B \rightarrow B$. In particular, $\phi_{\sigma}$ is an isogeny, but it is not guaranteed to be a compatible one. However, the map $\varphi \mapsto \phi_{\sigma}{ }^{\sigma} \varphi^{\circ} \phi_{\sigma}^{-1}: \mathcal{B} \rightarrow \mathcal{B}$ is a $\mathbb{Q}$-algebra automorphism of $\mathcal{B}$, so by the Noether-Skolem theorem it is inner: there exists a $\psi_{\sigma} \in \mathcal{B}^{\times}$ such that $\phi_{\sigma}{ }^{\sigma} \varphi^{\circ} \phi_{\sigma}^{-1}=\psi_{\sigma}^{-1} \circ \varphi \circ \psi_{\sigma}$. Since $\psi_{\sigma}$ is uniquely determined up to multiplication by rational numbers, we can choose $\psi_{\sigma}$ such that $\mu_{\sigma}=\psi_{\sigma} \circ \phi_{\sigma}$ is a compatible isogeny. In particular, if $k_{C}=\mathbb{Q}$ then $B$ is a building block.

\footnotetext{
${ }^{1}$ Recall that a polarization $\rho$ for $B$ is an isogeny $\rho: B \rightarrow \hat{B}$, and that the pullback of $\rho$ by $\phi_{\sigma}$ is $\phi_{\sigma}^{*}(\rho)=\hat{\phi}_{\sigma}^{\circ} \rho^{\circ} \phi_{\sigma}$.
} 
Recall that the degree of a compatible isogeny $\mu_{\sigma}$ is defined to be $d\left(\mu_{\sigma}\right)=$ $\mu_{\sigma} \circ \rho^{-1} \circ \hat{\mu}_{\sigma} \circ \rho$, which in our case can be identified with a rational number since the center of $\mathcal{B}$ is equal to $\mathbb{Q}$. The map

$$
\begin{array}{clc}
d: \operatorname{Gal}\left(\overline{\mathbb{Q}} / k_{C}\right) & \rightarrow & \mathbb{Q}^{\times} /\{ \pm 1\} \mathbb{Q}^{\times 2} \\
\sigma & \mapsto d\left(\mu_{\sigma}\right) \cdot\{ \pm 1\} \mathbb{Q}^{\times 2}
\end{array}
$$

is a homomorphism, and it gives the degree component $\overline{\left[c_{B}\right]}$ of $\left[c_{B}\right]$ under (the restriction of) the isomorphism (2.1). We will use the following notation to indicate elements in $\operatorname{Hom}\left(\operatorname{Gal}\left(\overline{\mathbb{Q}} / k_{C}\right), \mathbb{Q}^{\times} /\{ \pm 1\} \mathbb{Q}^{\times 2}\right)$ : if $t \in k_{C}$ and $\delta \in$ $\mathbb{Q}^{\times}$we denote by $(t, \delta)_{P}$ the homomorphism that sends $\sigma \in \operatorname{Gal}\left(\overline{\mathbb{Q}} / k_{C}\right)$ to $\delta \cdot\{ \pm 1\} \mathbb{Q}^{\times 2}$ if $\sigma \sqrt{t}=-\sqrt{t}$, and that sends $\sigma$ to the trivial element if $\sigma \sqrt{t}=\sqrt{t}$. Observe that any element of $\operatorname{Hom}\left(\operatorname{Gal}\left(\overline{\mathbb{Q}} / k_{C}\right), \mathbb{Q}^{\times} /\{ \pm 1\} \mathbb{Q}^{\times 2}\right)$ can be expressed (in a non-unique way) as a product of morphisms of the form $(t, \delta)_{P}$.

Proposition 3.2. Let $C$ be a curve as in Theorem 3.1, and let $d$ be the degree map associated to its Jacobian $B$. Then $d(\sigma) \equiv 1 \bmod \{ \pm 1\} \mathbb{Q}^{\times 2}$ for all $\sigma \in \operatorname{Gal}\left(\overline{\mathbb{Q}} / k_{\mathcal{O}}\right)$. If $\sigma \in \operatorname{Gal}\left(\overline{\mathbb{Q}} / k_{\mathbb{Z}\left[\omega_{h}\right]}\right)$ does not fix $k_{\mathcal{O}}$, then $d(\sigma) \equiv h$ $\bmod \{ \pm 1\} \mathbb{Q}^{\times 2}$, for $h \in\{m, D / m\}$.

Proof. Let $\sigma$ be an element in $\operatorname{Gal}\left(\overline{\mathbb{Q}} / k_{\mathcal{O}}\right)$, and let $\rho$ be the polarization on $B$ given by $C$. By the definition of the field of moduli there exists a compatible isomorphism $\phi_{\sigma}:{ }^{\sigma} B \rightarrow B$ such that $\phi_{\sigma}^{*}(\rho)={ }^{\sigma} \rho$. If we use $\rho$ to compute the degree we find that

$$
d\left(\phi_{\sigma}\right)=\phi_{\sigma} \circ \rho^{-1} \circ \hat{\phi}_{\sigma} \circ \rho=\phi_{\sigma} \circ \phi_{\sigma}^{-1} \circ \rho^{-1} \circ \hat{\phi}_{\sigma}^{-1} \circ \hat{\phi}_{\sigma} \circ \rho=1 .
$$

Let $\sigma$ be an element in $\operatorname{Gal}\left(\overline{\mathbb{Q}} / k_{\mathbb{Z}\left[\omega_{h}\right]}\right)$ that does not fix $k_{\mathcal{O}}$. By the definition of $k_{\mathbb{Z}\left[\omega_{h}\right]}$ there exists an isomorphism $\phi_{\sigma}:{ }^{\sigma} B \rightarrow B$ compatible with the endomorphisms in $\mathbb{Z}\left[\omega_{h}\right]$. By what we said above, there exists an element $\psi_{\sigma} \in \mathcal{B}$ such that $\mu_{\sigma}=\psi_{\sigma} \circ \phi_{\sigma}$ is an isogeny that is compatible with all the endomorphisms of $B$. The $\psi_{\sigma}$ satisfies that $\phi_{\sigma}{ }^{\sigma}{ }^{\sigma}{ }^{\circ} \phi_{\sigma}^{-1}=\psi_{\sigma}^{-1} \circ \varphi \circ \psi_{\sigma}$ for all $\varphi \in \mathcal{B}$, and if $\varphi$ belongs to $\mathbb{Z}\left[\omega_{h}\right]$ this implies that $\varphi=\psi_{\sigma}^{-1} \circ \varphi \circ \psi_{\sigma}$. Therefore, $\psi_{\sigma}$ commutes with every element in $\mathbb{Z}\left[\omega_{h}\right]$, which implies that $\psi_{\sigma}$ belongs to $\mathbb{Z}\left[\omega_{h}\right] \otimes \mathbb{Q}$. Hence, we have that $\psi_{\sigma}=a+b \omega_{h}$ for some $a, b \in \mathbb{Q}$ with $b \neq 0$. Indeed, if $b$ was 0 then $\psi_{\sigma}$ would be compatible with all the endomorphisms of $B$, but since $\sigma$ does not fix $k_{\mathcal{O}}$ this is not possible. Again, using $\rho$ to compute the degree of $\mu_{\sigma}$ we find that

$$
\begin{aligned}
d\left(\mu_{\sigma}\right) & =d\left(\psi_{\sigma} \circ \phi_{\sigma}\right)=\psi_{\sigma} \circ \phi_{\sigma}{ }^{\sigma} \rho^{-1} \circ \widehat{\psi_{\sigma} \circ \phi_{\sigma}} \rho \\
& =\psi_{\sigma} \circ \phi_{\sigma} \circ \phi_{\sigma}^{-1} \circ \rho^{-1} \circ \hat{\phi}_{\sigma}^{-1} \circ \hat{\phi}_{\sigma} \circ \hat{\psi}_{\sigma} \circ \rho \\
& =\psi_{\sigma} \circ \rho^{-1} \circ \hat{\psi}_{\sigma} \circ \rho=\psi_{\sigma} \circ \psi_{\sigma}^{\prime} .
\end{aligned}
$$


But the Rosati involution of an endomorphism $\varphi$ is given by $\varphi^{\prime}=\mu^{-1} \bar{\varphi} \mu$, and therefore

$$
\begin{aligned}
d\left(\mu_{\sigma}\right) & =\psi_{\sigma} \circ \psi_{\sigma}^{\prime}=\left(a+b \omega_{h}\right)\left(a+b \omega_{h}\right)^{\prime}=\left(a+b \omega_{h}\right) \mu^{-1}\left(a-b \omega_{h}\right) \mu \\
& =\left(a+b \omega_{h}\right)^{2}=a^{2}+h b^{2}+2 a b \omega_{h} .
\end{aligned}
$$

The degree $d\left(\mu_{\sigma}\right)$ belongs to $\mathbb{Q}^{\times}$, and since $b \neq 0$ then necessarily $a=0$ and $d\left(\mu_{\sigma}\right) \equiv h\left(\bmod \mathbb{Q}^{\times 2}\right)$.

When $B$ is a building block, we can use this knowledge of the degree map to compute $\left[c_{B}\right]$.

Proposition 3.3. Suppose that $k_{C}=\mathbb{Q}$, and let $k_{\mathbb{Z}\left[\omega_{h}\right]}=\mathbb{Q}\left(\sqrt{t_{h}}\right)$ for $h \in\{m, D / m\}$. The sign and degree components of $\left[c_{B}\right]$ are given by

$$
\begin{gathered}
\overline{\left[c_{B}\right]}=\left(t_{m}, D / m\right)_{P} \cdot\left(t_{D / m}, m\right)_{P} \\
{\left[c_{B}\right]_{ \pm}=\left(t_{m}, D / m\right)_{\mathbb{Q}} \cdot\left(t_{D / m}, m\right)_{\mathbb{Q}} \cdot(-D, m)_{\mathbb{Q}} .}
\end{gathered}
$$

Proof. The expression for the degree component follows from Proposition 3.2. First of all, the degree homomorphism $d$ is the inflation of a homomorphism from $\operatorname{Gal}\left(k_{\mathcal{O}} / \mathbb{Q}\right)$, and we know that $k_{\mathcal{O}}=\mathbb{Q}\left(\sqrt{t_{m}}, \sqrt{t_{D / m}}\right)$. Note that we are not assuming this to be a degree 4 extension: it can also be a quadratic or a trivial extension. Let $\sigma \in \operatorname{Gal}\left(k_{\mathcal{O}} / k_{\mathbb{Z}\left[\omega_{m}\right]}\right)$ that does not fix $k_{\mathcal{O}}$; it restricts to a generator of $\operatorname{Gal}\left(\mathbb{Q}\left(\sqrt{t_{D / m}}\right) / \mathbb{Q}\right)$, and as we have seen $d(\sigma) \equiv m \bmod \{ \pm 1\} \mathbb{Q}^{\times 2}$. This gives the part $\left(t_{D / m}, m\right)_{P}$ of the degree homomorphism. In the same way, if $\tau$ is an element from $\operatorname{Gal}\left(k_{\mathcal{O}} / k_{\mathbb{Z}\left[\omega_{D / m}\right]}\right)$ that does not fix $k_{\mathcal{O}}$, it restricts to a generator of $\operatorname{Gal}\left(\mathbb{Q}\left(\sqrt{t_{m}}\right) / \mathbb{Q}\right)$ and $d(\sigma) \equiv D / m \bmod \{ \pm 1\} \mathbb{Q}^{\times 2}$, which gives the part $\left(t_{D / m}, m\right)_{P}$.

Now, to prove the identity (3.2) we use [7, Theorem 2.8], which gives a formula for the Brauer class of the endomorphism algebra of a building block. Particularized to our case, and having computed the degree component, this formula gives

$$
(-D, m)_{\mathbb{Q}}=\left[c_{B}\right]_{ \pm} \cdot\left(t_{m}, D / m\right)_{\mathbb{Q}} \cdot\left(t_{D / m}, m\right)_{\mathbb{Q}}
$$

Here $H^{2}\left(G_{\mathbb{Q}},\{ \pm 1\}\right)$ is identified with the 2-torsion of the Brauer group of $\mathbb{Q}$. From this (3.2) follows immediately.

\section{A concrete example}

Let $\mathcal{B}_{6}=(2,3)_{\mathbb{Q}}$ be the rational quaternion algebra of discriminant 6 . Let $\imath, \jmath$ be elements in $\mathcal{B}_{6}$ such that $\imath^{2}=2$ and $\jmath^{2}=3$, and let $\mu=2 \jmath+\imath$. The order $\mathcal{O}=\mathbb{Z}[\imath,(1+\jmath) / 2]$ is maximal, and the elements $\omega_{2}=\imath$ and $\omega_{3}=\jmath+\imath \jmath$ are twists of $(\mathcal{O}, \mu)$. For ease of notation, we define the subrings $R_{2}=\mathbb{Z}[\imath] \simeq \mathbb{Z}[\sqrt{2}], R_{3}=\mathbb{Z}[\jmath+\imath \jmath] \simeq \mathbb{Z}[\sqrt{-3}]$ and $R_{6}=\mathbb{Z}[\mu] \simeq \mathbb{Z}[\sqrt{6}]$ 
We will consider the following genus two curve:

$$
\begin{aligned}
C: \quad Y^{2}= & (-4+2 \sqrt{2}) X^{6}-12^{2} X^{5}-72(28+2 \sqrt{2}) X^{4}+16 \cdot 12^{2} X^{3} \\
& +12^{3}(28-6 \sqrt{2}) X^{2}-48 \cdot 12^{3} X+8 \cdot 12^{3}(4+2 \sqrt{2}) .
\end{aligned}
$$

It has been obtained by particularizing to $-4 / 27$ the value of the parameter $j$ in the family of curves described in [1, Theorem 15]. Let $B$ be the Jacobian of $C$, which is a polarized abelian surface (with the canonical polarization given by $C$ ). The results on the arithmetic of such curves of $[1, \S 3.6]$ give us the following information about $B$ :

Proposition 4.1 (Baba-Granath). The endomorphism algebra of $B$ is isomorphic to $\mathcal{B}_{6}$, and under this isomorphism the Rosati involution attached to the canonical polarization of $B$ is given by $\varphi^{\prime}=\mu^{-1} \bar{\varphi} \mu$. The several fields of moduli are $k_{\mathbb{Z}}=\mathbb{Q}, k_{R_{3}}=\mathbb{Q}$ and $k_{\mathcal{O}}=k_{R_{2}}=k_{R_{6}}=\mathbb{Q}(\sqrt{-3})$. Finally, all the endomorphisms of $B$ are defined over $K=\mathbb{Q}(\sqrt{2}, \sqrt{-3})$.

If $\sigma \in G_{\mathbb{Q}}$ restricts to the non-trivial automorphism of $\mathbb{Q}(\sqrt{2}) / \mathbb{Q}$ then the map $(x, y) \mapsto\left(\frac{-24}{x}, \frac{(-24)^{3 / 2} y}{x^{3}}\right)$ is an isomorphism ${ }^{\sigma} C \rightarrow C$, that gives rise to an isomorphism ${ }^{\sigma} B \simeq B$ defined over $K$. Therefore, $B$ is a building block completely defined over $K$.

Proposition 4.2. The sign and degree components of $\left[c_{B}\right]$ are $\overline{\left[c_{B}\right]}=$ $(-3,3)_{P}$ and $\left[c_{B}\right]_{ \pm}=(-6,3)_{\mathbb{Q}}$.

Proof. This is a direct application of Proposition 3.3. Indeed, Proposition 4.1 directly gives that in this case $t_{2}=-3$ and $t_{3}=1$.

We will use this result to see that $\left[c_{B / K}\right]$ is not symmetric. As in (2.1) we have a decomposition

$$
H^{2}\left(G, \mathbb{Q}^{\times}\right) \simeq H^{2}(G,\{ \pm 1\}) \times \operatorname{Hom}\left(G, \mathbb{Q}^{\times} /\{ \pm 1\} \mathbb{Q}^{\times 2}\right),
$$

where $G=\operatorname{Gal}(K / \mathbb{Q})$ acts trivially on $\mathbb{Q}^{\times}$; we denote by $\left[c_{B / K}\right]_{ \pm}$and $\overline{\left[c_{B / K}\right]}$ respectively the components of $\left[c_{B / K}\right]$ under this identification. Since $G$ is abelian, the component $\overline{\left[c_{B / K}\right]}$ is always symmetric, so we are only concerned with the symmetry of the component $\left[c_{B / K}\right]_{ \pm}$.

The groups $H^{2}(G,\{ \pm 1\})$ and $\operatorname{Ext}(G,\{ \pm 1\})$ have dimension 3 and 2 respectively as $\mathbb{Z} / 2 \mathbb{Z}$-vector spaces, and we will write down explicit bases for them (the reader can consult $[6, \S 2]$ for proofs of the following statements related to these bases). If $\varepsilon: G \rightarrow\{ \pm 1\}$ is a character, for each $\sigma \in G$ fix a square root $\sqrt{\varepsilon(\sigma)}$ and let $c_{\varepsilon}: G \times G \rightarrow\{ \pm 1\}$ be the map

$$
c_{\varepsilon}(\sigma, \tau)=\sqrt{\varepsilon(\sigma)} \sqrt{\varepsilon(\tau)} \sqrt{\varepsilon(\sigma \tau)}^{-1} .
$$

It is a 2-cocycle and its cohomology class does not depend on the choice of the square roots. Let $\varepsilon_{2}$ denote the character $G \rightarrow\{ \pm 1\}$ with kernel 
$\operatorname{Gal}(K / \mathbb{Q}(\sqrt{2}))$, and let $\varepsilon_{-3}$ be the character with kernel $\operatorname{Gal}(K / \mathbb{Q}(\sqrt{-3}))$. Then a basis of $\operatorname{Ext}(G,\{ \pm 1\})$ is given by $\left\{\left[c_{\varepsilon_{2}}\right],\left[c_{\varepsilon_{-3}}\right]\right\}$. Denote by $\chi_{2}$ and $\chi_{-3}$ the additive version of the characters $\varepsilon_{2}$ and $\varepsilon_{-3}$ (i.e. the same characters but viewed as taking values in $\{0,1\})$, and define a cocycle $c_{2,-3}$ by the formula

$$
c_{2,-3}(\sigma, \tau)=(-1)^{\chi_{2}(\sigma) \chi_{-3}(\tau)} .
$$

A basis for $H^{2}(G,\{ \pm 1\})$ is then given by $\left\{\left[c_{\varepsilon_{2}}\right],\left[c_{\varepsilon_{-3}}\right],\left[c_{2,-3}\right]\right\}$. The inflation of these cohomology classes to $H^{2}\left(G_{\mathbb{Q}},\{ \pm 1\}\right) \simeq \operatorname{Br}(\mathbb{Q})[2]$ is as follows: $\operatorname{Inf}\left(\left[c_{\varepsilon_{2}}\right]\right)=(2,-1)_{\mathbb{Q}}, \operatorname{Inf}\left(\left[c_{\varepsilon_{-3}}\right]\right)=(-3,-1)_{\mathbb{Q}}$ and $\operatorname{Inf}\left(\left[c_{2,-3}\right]\right)=(2,-3)_{\mathbb{Q}}$.

We know that $\operatorname{Inf}\left(\left[c_{B / K}\right]_{ \pm}\right)=(-6,3)_{\mathbb{Q}}$, and it is easy to check that the only elements in $H^{2}(G,\{ \pm 1\})$ whose inflation is $(-6,3)_{\mathbb{Q}}$ are $\left[c_{2,-3}\right]$ and $\left[c_{\varepsilon_{2}}\right] \cdot\left[c_{2,-3}\right]$; this means that $\left[c_{B / K}\right]_{ \pm}=\left[c_{2,-3}\right]$ or $\left[c_{B / K}\right]_{ \pm}=\left[c_{\varepsilon_{2}}\right] \cdot\left[c_{2,-3}\right]$. Since neither of the two options belong to $\operatorname{Ext}(G,\{ \pm 1\})$ we see that $\left[c_{B / K}\right]_{ \pm}$ is not symmetric. This means that $B$ is not strongly modular over $K$; what is more, no variety $\overline{\mathbb{Q}}$-isogenous to $B$ is strongly modular over $K$.

Let $L=K(\sqrt{-1})$ and let $\gamma=\sqrt{6}+\sqrt{18}$ which is in $L$. Let $C_{\gamma}$ be the quadratic twist of $C$ :

$$
\begin{aligned}
C_{\gamma}: \quad \gamma Y^{2}= & (-4+2 \sqrt{2}) X^{6}-12^{2} X^{5}-72(28+2 \sqrt{2}) X^{4}+16 \cdot 12^{2} X^{3} \\
& +12^{3}(28-6 \sqrt{2}) X^{2}-48 \cdot 12^{3} X+8 \cdot 12^{3}(4+2 \sqrt{2}) .
\end{aligned}
$$

and denote by $B_{\gamma}$ its Jacobian. Now we can use [3, Lemma 6.1] to see that it is strongly modular over $L$. First of all, since $L / \mathbb{Q}$ is Galois, we see that $B_{\gamma}$ is a building block also completely defined over $K$. Moreover, the cohomology class $\left[c_{B_{\gamma} / L}\right]$ is the product of $\left[c_{B / L}\right]$ with the cohomology class in $H^{2}(\operatorname{Gal}(L / \mathbb{Q}),\{ \pm 1\})$ associated to the exact sequence

$$
1 \rightarrow \operatorname{Gal}(L(\sqrt{\gamma}) / L) \simeq\{ \pm 1\} \rightarrow \operatorname{Gal}(L(\sqrt{\gamma}) / \mathbb{Q}) \rightarrow \operatorname{Gal}(L / \mathbb{Q}) \rightarrow 1 .
$$

The cohomology class attached to this exact sequence turns out to be equal to $\left[c_{\varepsilon_{-1}}\right] \cdot\left[c_{\varepsilon_{-3}}\right] \cdot\left[c_{\varepsilon_{-6}}\right] \cdot\left[c_{2,-3}\right]$. This implies that $\left[c_{B_{\gamma} / L}\right]$ is symmetric, because the factor $\left[c_{2,-3}\right]$ vanishes. In conclusion, $B_{\gamma}$ is strongly modular over $L$, so $L\left(B_{\gamma} / L ; s\right)$ is equivalent to a product of $L$-series of newforms for $\Gamma_{1}(N)$.

We want to find the newforms the product of whose $L$-series is the $L$ function of the variety $B_{\gamma} / L$. Since the curve $C_{\gamma}$ has good reduction for primes different from 2 and 3 we must look for newforms of level a product of a power of two and a power of three. Using Magma [4] we found a modular form $f \in S_{2}\left(\Gamma_{1}\left(2^{4} \cdot 3^{4}\right), \chi\right)$, where $\chi$ is the quadratic character of conductor 12, whose Fourier expansion begins with

$$
\begin{aligned}
f= & q-\sqrt{3} q^{5}+3 \sqrt{-1} q^{7}-3 \sqrt{3} q^{11}+q^{13}-2 \sqrt{-3} q^{17}-6 \sqrt{-1} q^{19} \\
& +3 \sqrt{3} q^{23}+2 q^{25}-5 \sqrt{-3} q^{29}-3 \sqrt{-1} q^{31}+\cdots
\end{aligned}
$$


and a modular form $g \in S_{2}\left(\Gamma_{1}\left(2^{6} \cdot 3^{4}\right), \chi\right)$ whose Fourier expansion begins with:

$$
\begin{aligned}
g= & q-\sqrt{3} q^{5}+3 \sqrt{-1} q^{7}-3 \sqrt{3} q^{11}-q^{13}+2 \sqrt{-3} q^{17}+6 \sqrt{-1} q^{19} \\
& -3 \sqrt{3} q^{23}+2 q^{25}-5 \sqrt{-3} q^{29}-3 \sqrt{-1} q^{31}+\cdots .
\end{aligned}
$$

We computed the $p$-th Euler factor of $L\left(B_{\gamma} / L ; T\right)$ and checked the identity

$$
L_{p}\left(B_{\gamma} / L ; T\right)=L_{p}\left(A_{f} ; T\right)^{2} \cdot L_{p}\left(A_{g} ; T\right)^{2}
$$

for all primes $p<1000, p \neq 2,3$. Here, the $L$-series of $A_{f}$ means the product of the $L$-series of the Galois conjugate forms of $f$, and similarly for $g$. We also checked that no modular forms of smaller levels produce identities of this type.

These numerical verifications give a good checking of the correctness of the computations in the paper and also provide a near certainty of the equivalence of $L$-series

$$
L\left(B_{\gamma} / L ; s\right) \sim L\left(A_{f} ; s\right)^{2} \cdot L\left(A_{g} ; s\right)^{2},
$$

although in order to have a complete proof of this equivalence one should compute the conductor of $B_{\gamma} / L$ in order to bound the levels of the forms to look for identities, and the standard mathematical software available cannot perform that computation.

\section{References}

[1] S. Baba, H. Granath, Genus 2 curves with quaternionic multiplication. Canad. J. Math. 60 (2008), no. 4, 734-757.

[2] J. GonzÁlez, J.-C. LARio, $\mathbb{Q}$-curves and their Manin ideals. Amer. J. Math. 123 (2001), no. 3, 475-503.

[3] X. Guitart, J. Quer, Modular abelian varieties over number fields. Submitted. Preprint available at http://arxiv.org/abs/0905.2550v1

[4] J. J. Cannon, W. Bosma (Eds.), Handbook of Magma Functions, Edition 2.15-13 (2009).

[5] E. E. PyLE, Abelian varieties over $\mathbb{Q}$ with large endomorphism algebras and their simple components over $\overline{\mathbb{Q}}$. Modular curves and abelian varieties, Progress in Math., vol. 224 (2002), pp. 189-239.

[6] J. QUER, Embedding problems over abelian groups and an application to elliptic curves. J. Algebra 237 (2001), no. 1, pp. 186-202.

[7] J. Quer, Fields of definition of building blocks. Math. Comp. 78 (2009), pp. 537-554.

[8] K. A. RIBet, Abelian varieties over $\mathbb{Q}$ and modular forms. Modular curves and abelian varieties, Progress in Math., vol. 224 (2002), pp. 241-261.

[9] K. A. RiBet, Twists of modular forms and endomorphisms of abelian varieties. Math. Ann. 253 (1980), no. 1, 43-62.

[10] V. Rotger, The field of moduli of quaternionic multiplication on abelian varieties. Int. J. Math. Math. Sci. 2004, no. 49-52, 2795-2808.

[11] A. WeIL, Adeles and algebraic groups. With appendices by M. Demazure and Takashi Ono. Progress in Mathematics, 23. Birkhuser, Boston, Mass., 1982. iii+126 pp. 


\section{Xavier GuitarT}

Dept. Matemàtica Aplicada II

Universitat Politècnica de Catalunya

Carrer Colom 11

08222 Terrassa

E-mail: xevi.guitart@gmail.com

\section{Jordi QueR}

Dept. Matemàtica Aplicada II

Universitat Politècnica de Catalunya

Carrer Jordi Girona 1-3

08028 Barcelona

E-mail: jordi.quer@upc.edu 